Pathologe 2008 · [Suppl 2] 29:286-289 DOI 10.1007/s00292-008-1087-y

Online publiziert: 28. September 2008

๑) Springer Medizin Verlag 2008

H.P. Dienes - U. Drebber

Kompetenznetzwerk HepNet, Deutsche Leberstiftung, Institut für Pathologie, Universitätsklinikum Köln

\title{
Aktuelle Probleme der Hepatitis
}

Tab. 1 Histopathologie der chroni-

schen Hepatitis C: Zusammenfassung

der wichtigsten histologischen Veränderungen. (Nach [16])

\begin{tabular}{ll}
\hline Histologische Merkmale & $\begin{array}{l}\text { Häufigkeit } \\
{[\%]}\end{array}$ \\
\hline $\begin{array}{l}\text { Lymphoide Follikel } \\
80\end{array}$ & 70 \\
\hline $\begin{array}{l}\text { Makrovesikuläre Steatose } \\
\begin{array}{l}\text { Auffällige Aktivation } \\
\text { sinusoidaler Zellen }\end{array}\end{array}$ & 60 \\
\hline $\begin{array}{l}\text { Läsionen des Gallengangs } \\
\text { Mallory-Körper }\end{array}$ & 30 \\
\hline
\end{tabular}

Tab. 2 Zusammenfassung der Rolle der relevanten histologischen Verände-

rungen zur Erstellung des Scores für die

autoimmune Hepatitis (AIH).

(Nach Empfehlung von Alvarez et al. [1])

Parameter/Merkmale Score

Drogenkonsum

positiv $\quad-4$

negativ $\quad+1$

Durchschnittlicher Alkoholkonsum

$<25 \mathrm{~g} / \mathrm{Tag}+2$

$>60 \mathrm{~g} / \mathrm{Tag} \quad-2$

Leberhistologie

Interface-Hepatitis +3

hauptsächlich lymphoplasma- $\quad+1$

zytisches Infiltrat

Rosettierung der Leberzellen $\quad+1$

keines der oben genannten $\quad-5$

biliäre Veränderungen -3

andere Veränderungen -3

andere Autoimmunkrankheit(en) +2

Weitere Parameter (optional)

Seropositivität für andere, $\quad+2$

definierte Antikörper

HLA-DR3 oder -DR4

$+1$

Therapieansprechen

komplett

Rezidiv $+3$
In den letzten Jahren hat sich das Bild der Virushepatitis, bedingt durch den raschen Fortschritt in der molekularen Virologie, erheblich geändert. Es wurden verschiedene neue Formen der Hepatitis heraus gearbeitet, die einer speziellen Diagnostik vor allem mit den modernen Mitteln der Molekularbiologie bedürfen, wobei diese auch am Paraffinmaterial der Biopsien angewendet werden können. Im Folgenden sollen die neuen Erkenntnisse auf dem Gebiet der Virushepatitiden B, C, D und $\mathrm{E}$ sowie bei Erkrankungen mit nichthepatotropen Viren, wie Epstein-Barr-Virus (EBV) und Zytomegalievirus (CMV), besprochen werden.

Für die Diagnose der autoimmunen Hepatitis wurden kürzlich neue Kriterien vorgeschlagen, die hier kurz eingeführt und kommentiert werden.

\section{Hepatitis B}

Der natürliche Verlauf der chronischen Hepatitis B wird inzwischen besser verstanden, weil die modernen molekularbiologischen Methoden noch einzelne Virusgenome nachweisen können und das Schicksal des Virus im Organismus inzwischen so beschrieben wird, dass mit dem Fortgang der Erkrankung die Menge an replizierenden Viren allmählich und kontinuierlich abnimmt. Es gibt also eine späte Phase, in der das Virus im Serum unter die Nachweisgrenze fallen kann. Dennoch haben die Patienten weiterhin eine chronische Hepatitis B, die jederzeit unter dem Einfluss exogener Faktoren oder einer Schwäche des Immunsystems wieder aufflammen kann. Diese Phase wird als okkulte Hepatitis B bezeichnet, von Tor- benson [12] zum ersten Mal so benannt. Diese Definition wird zurzeit neu überarbeitet. Die Form der okkulten Hepatitis B hat insofern eine erhebliche klinische Bedeutung, als diese Patienten unter dem Einfluss von Zytostatika oder Immunsuppressiva einen massiven Schub einer Hepatitis B entwickeln können, möglicherweise mit fatalen Folgen.

Die Histopathologie dieser Patienten mit okkulter Hepatitis B kann sehr unterschiedlich sein und reicht von einer milden Entzündung (Grad II) über die Leberfibrose, Leberzirrhose bis hin zum hepatozellulären Karzinom [14] mit HBx-Antigen als dem einzigen Virusmarker im Gewebe $[3,11]$. Bei Verdacht auf das Vorliegen einer okkulten Hepatitis B mit negativen Resultaten im Serum sollte versucht werden, mit Hilfe einer Polymerasekettenreaktion (PCR) am Gewebe das Virusgenom direkt in der Leber darzustellen.

\section{Hepatitis C}

Bei der chronischen Hepatitis C ist inzwischen die Erkenntnis gesichert, dass der Leberschaden (• Tab. 1) nicht nur durch das Virus selbst, bzw. durch die Immunantwort gegen das Virus, hervorgerufen wird, sondern es kann zu zusätzlichen Schäden der Leber durch metabolische Entgleisungen kommen, bei denen das Virus jedoch eine ursächliche Rolle spielt. Im Einzelnen betrifft dies den Fettstoffwechsel und den Eisenstoffwechsel mit Eisenüberladung des Lebergewebes.

Zwischen Virus, Virusreplikation und Fettstoffwechsel der Leber besteht ein enges intrinsisches Verhältnis, da das Virus für seine Replikation feinste Fetttropfen 
Abb. 2 a Akute sporadische Hepatitis E eines deutschen Patienten: Man findet das Bild einer klassischen Virushepatitis, in diesem Falle mit deutlicher Cholestase (HE-Färbung, Vergr. 200:1). b Typischer Verlauf einer akuten Hepatitis E mit Darstellung der Laborwerte und des Virusnachweises
Abb. $1>$ Histologie einer chronischen Hepatitis C ohne Nachweis von HCV-RNA im Serum, jedoch im Lebergewebe. Charakteristisch ist die Verfettung bei nahezu rein lymphozytärer Infiltration
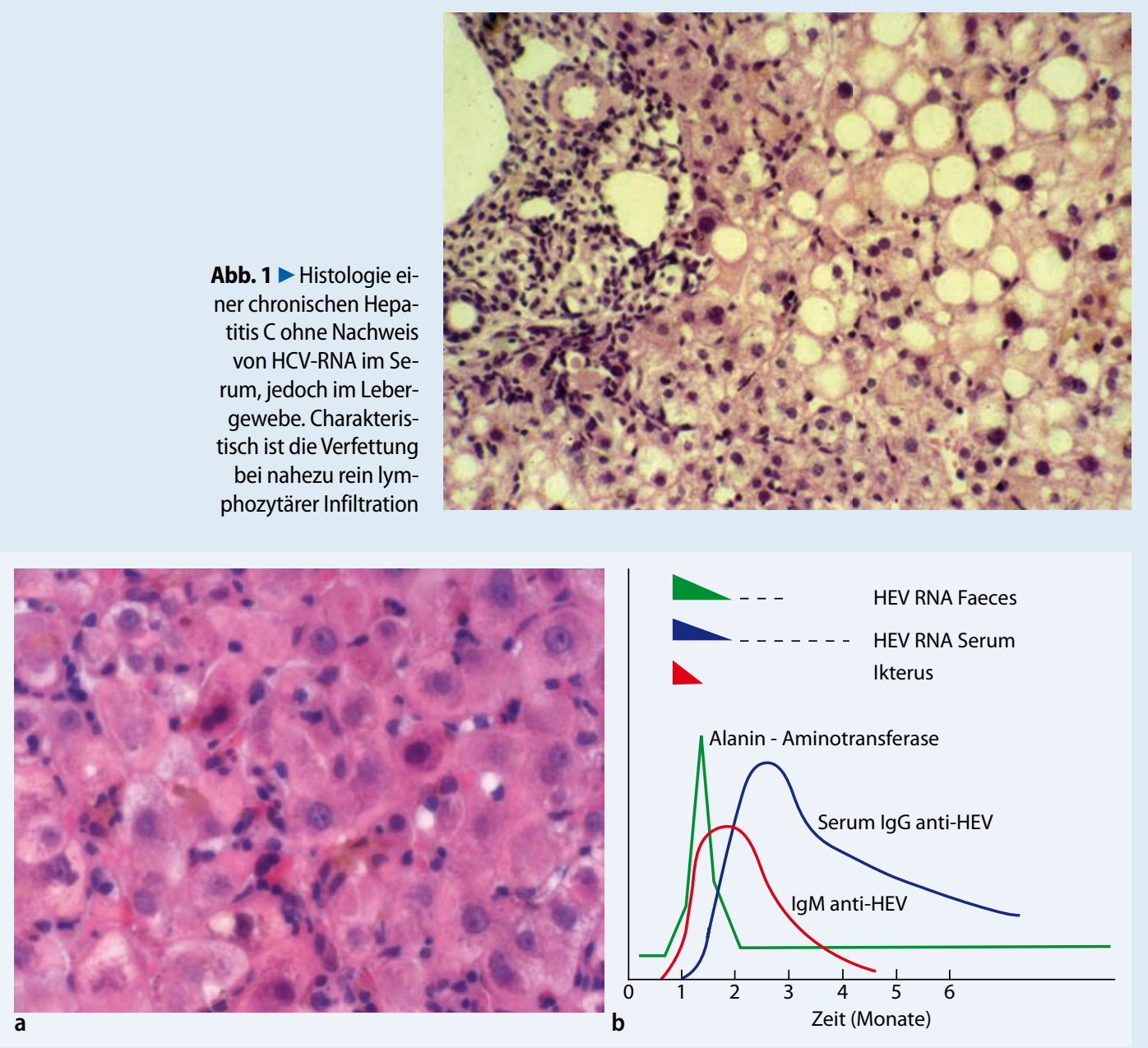

als Matrize für den Virusaufbau benötigt und molekulare Strukturen im HCV (Core, E, NS3- und -5-Region) direkt in den Fettstoffwechsel eingreifen können. Die Formen der Mechanismen hängen zudem vom Genotyp des Virus ab, und der Typ 3 des HCV besitzt die Fähigkeit, direkt zur Steatose der Leber zu führen. Die häufigeren Virustypen $1 \mathrm{a}$ und $1 \mathrm{~b}$ dagegen induzieren eine Steatosis über andere Mechanismen [2], die dann eine eigenständige Dynamik entwickeln. Eine Steatohepatitis im Rahmen einer zugrunde liegenden chronischen Hepatitis C verläuft dann nach den intrinsischen Mechanismen einer Steatohepatitis ab.

Es hat sich gezeigt, dass die Steatose bei der chronischen Hepatitis C ( $\bullet$ Abb. 1) ein relevanter Risikofaktor für eine beschleunigte Progression der Erkrankung ist und auch das Risiko für die Entstehung eines Leberzellkarzinoms deutlich erhöht. Es konnte auch demonstriert werden, dass das Virus in die Pathophysiologie der Adipokine eingreift, und Patienten mit chronischer Hepatitis C lassen einen verringerten Spiegel von Adiponektin [4] im Serum erkennen $[4,10]$. Wir selbst haben Polymorphismen im Exon 2 des Adiponektin-Gens untersucht und gefunden, dass Patienten mit einer chronischen $\mathrm{He}$ patitis C und Steatohepatitis mit Entzündungsgrad 3 bis 4 Polymorphismen in diesem Lokus aufweisen [13].

Schon früh in der klinischen Erforschung der chronischen Hepatitis C hatte sich gezeigt, dass viele Patienten eine verstärkte Eisenablagerung in der Leber aufweisen, und man hatte auch früh erkannt, dass die Eisenüberladung der Leber bei einem Patienten mit einer chronischen Hepatitis C die Antwort auf eine Therapie mit Interferon deutlich verschlechtert. In den letzten Jahren haben sich zwei Mechanismen herauskristallisiert, die zu einer Eisenüberladung der Leber im Ver- lauf einer chronischen Hepatitis C führen können:

- Zum einen kann das Virus den Transferrinrezeptor 2 an der Leberzellmembran hoch regulieren, sodass vermehrt Eisen in die Zelle aufgenommen wird [8].

- Zum anderen konnte gezeigt werden, dass das Protein Hepcidin, das die Aufnahme von Eisen in die Zelle gegenreguliert, durch das HCV gehemmt wird [6].

\section{Hepatitis D}

Das Hepatitis-D-Virus (HDV) führt auch in Mitteleuropa immer noch zu zahlreichen Koinfektionen mit HBV, was sehr wahrscheinlich auf die Immigrantenbewegungen zurückzuführen ist [15]. Das Virus führt zu einer schwereren und progredienteren Form der Hepatitis als HBV alleine. Wir haben ein Patientenkollektiv 
Pathologe 2008 · [Suppl 2] 29:286-289

DOI 10.1007/s00292-008-1087-y

(c) Springer Medizin Verlag 2008

\section{H.P. Dienes · U. Drebber}

\section{Aktuelle Probleme der Hepatitis}

\section{Zusammenfassung}

Bei den verschiedenen Formen der Virushepatitis haben sich in den letzten Jahren hinsichtlich bestimmter Verlaufsformen, des ätiopathogenetischen Zusammenhangs von Komorbiditäten und der Beschreibung neuer Formen in Mitteleuropa neue Erkenntnisse ergeben.

Die Erkrankung durch nichthepatotrope Viren wie Epstein-Barr-Virus (EBV) und Zytomegalievirus (CMV) konnten in letzter Zeit aufgrund moderner molekularbiologischer Methoden mit direktem Virusgenomnachweis an in Paraffin eingebetteten Leberbiopsien besser beschrieben werden.

Für die Diagnose der autoimmunen Hepatitis wurden auf dem Boden einer großen Multicenter-Datenbank neue diagnostische Kriterien vorgeschlagen.

\section{Schlüsselwörter}

Virushepatitis - Hepatitis B - Hepatitis C.

Autoimmune Hepatitis

\section{Current problems of hepatitis}

\section{Abstract}

New findings have been made in recent years on the various forms of the hepatitis virus in terms of disease course, its etiopathogenetic link with comorbidities and the definition of new forms in Central Europe.

Epstein-Barr virus (EBV)- and cytomegalovirus (CMV)-induced hepatitis may occur in the so-called sero-negative group of hepatitis and direct demonstration of the viral genome in paraffin liver tissues is required to confirm the diagnosis.

Since diagnosis of autoimmune hepatitis in daily practice may be difficult, a scoring system with simplified criteria has recently been developed.

\section{Keywords}

Virushepatitis - Hepatitis B · Hepatitis C . Autoimmune hepatitis

Tab. 3 Tabelle der vereinfachten Kriterien zur Diagnosestellung einer autoimmunen Hepatitis (AlH). (Nach [7])

\begin{tabular}{|c|c|c|}
\hline Variable & Grenze & Punkte \\
\hline ANA oder SMA & $\geq 1: 40$ & 1 \\
\hline ANA oder SMA & $\geq 1: 80$ & \\
\hline oder LKM & $\geq 1: 40$ & $2^{\mathrm{a}}$ \\
\hline oder SLA & Positiv & \\
\hline \multirow[t]{2}{*}{$\lg G$} & >normale Wertobergrenze & 1 \\
\hline & $\begin{array}{l}>1,10 \text { fach normale Wertober- } \\
\text { grenze }\end{array}$ & 2 \\
\hline \multicolumn{3}{|l|}{ Leberhistologie } \\
\hline \multirow{2}{*}{$\begin{array}{l}\text { (Anzeichen einer Hepatitis ist } \\
\text { eine conditio sine qua non) }\end{array}$} & Kompatibel mit AlH & 1 \\
\hline & Typische AlH & 2 \\
\hline \multirow[t]{3}{*}{ Fehlende virale Hepatitis } & Ja & 2 \\
\hline & \multirow[t]{2}{*}{ Summe: } & $\geq 6$ : vermutlich $\mathrm{AlH}$ \\
\hline & & $\geq 7$ : definitiv AlH \\
\hline
\end{tabular}

von knapp 100 Patienten mit HBV- und HDV-Koinfektion untersucht, ScoringSysteme nach Ishak eingesetzt und mit anderen Daten und dem Krankheitsverlauf verglichen. Es zeigte sich, dass HDV-RNA keine Korrelation mit biochemischen $\mathrm{Pa}$ rametern oder der Histopathologie ergab, sodass HDV selbst nicht als zytopathisch für die Leberzellen anzusehen ist. Biochemische Parameter sind nicht in der Lage, Stadium und Grad der chronischen HDV-Infektion exakt zu bestimmen, sodass die Leberbiopsie unerlässlich für ein reales Bild des Ausmaßes der Leberschädigung ist.

\section{Hepatitis E}

Infektionen mit Hepatitis-E-Virus (HEV) werden in letzter Zeit in der Literatur auch als sporadische Fälle einer Hepatitis in Mitteleuropa gemeldet, obwohl man bislang annahm, dass die Erreger aus den Tropen oder Subtropen eingeschleppt wurden. Der Erreger, ein Einzelstrang-RNA-Virus ohne Hülle von $34 \mathrm{~nm}$ Durchmesser, wird inzwischen der Familie der Hepe-Viridae zugeordnet. Die Diagnose kann durch die Serologie erfolgen, wobei die derzeit verfügbaren kommerziellen Assays nicht sehr sensitiv sind. Der direkte Nachweis erfolgt durch RT-PCR. Die Infektion zeigt das Bild einer klassischen akuten Virushepatitis, oft mit cholestatischer Komponente (• Abb. 2 a, b). Die nekroinflammatorischen Merkmale zeigen eine portoperiportale Prädominanz im Läppchen.

\section{EBV-Hepatitis}

Das klassische Bild der EBV-Hepatitis ist seit Langem bekannt. Durch den Einsatz moderner sensitiver Nachweismethoden des Virusgenoms direkt am Gewebe hat sich jedoch gezeigt, dass es morphologische Varianten gibt, die neu zu beschreiben sind. Dieses ist vor allem der Fall bei der späten Erstinfektion oder bei Reaktivierung [5]. Das klassische Bild zeigt ein diffuses lymphozytäres Infiltrat in den Sinusoiden mit fokalen Apoptosen, wobei sich das Infiltrat aus infizierten B-Lymphozyten, aktivierten T-Lymphozyten und NK-Zellen zusammensetzt. Es kann zur Endothelitis kommen, ferner auch zur Cholangitis, und seltener sind Granulome und Steatosis. Einzelne Vorkommen von sogenannten Fibrinringgranulomen werden berichtet. In seltenen Fällen kann es auch zum Verlauf einer chronischen Hepatitis kommen. In unserem eigenen Untersuchungsgut von Fällen mit sogenannter seronegativer okkulter Hepatitis werden noch in 6\% der Biopsien EBV-Genome nachgewiesen [5].

\section{Autoimmune Hepatitis}

Die autoimmune Hepatitis (AIH) lässt sich in der Regel im klinischen Alltag gut diagnostizieren. Da es für die $\mathrm{AIH}$ je- 
doch keine spezifischen und sensitiven Autoantikörper als diagnostische Marker gibt, kann es in einem nicht unbeträchtlichen Prozentsatz zu Schwierigkeiten bei der Diagnosenabsicherung kommen. Im Jahre 1999 hat eine internationale Gruppe versucht, einen Diagnosescore für die AIH zu erstellen ([1]; • Tab. 2). Im Laufe der Jahre hat sich jedoch gezeigt, dass die Anwendung dieses Scores im klinischen Alltag Schwierigkeiten bereiten kann, und es wurden neue Formulierungen für die Absicherung der Diagnose gesucht. So war in dem alten Vorschlag die Leberhistologie beschrieben als: „Inter-Grenzflächenhepatitis vorwiegend lymphoplasmazelluläre Infiltrate und Rosettieren der Leberzellen“. Veränderungen an den Gallengängen wurden als nicht kompatibel betrachtet.

Inzwischen hat sich ein neues Konzept zur Diagnose der AIH durchgesetzt [7]. Bei diesem werden die histologischen Veränderungen mit dem Punktwert von „O“ eingesetzt, wenn die Histologie untypisch für eine AIH ist, z. B. Verfettung (- Tab. 3). Ein Punktwert von „“ "erfolgt, wenn die Veränderungen vereinbar mit einer AIH sind, und ein Punktwert von „2“ wird dann gegeben, wenn die Histologie typisch für eine AIH ist.

Die jüngste Literatur über die $\mathrm{AIH}$ hat die Betonung auf sogenannte „Varianten der AIH“ gelegt, die sich zusammenfassen lassen als [9]:

- seronegative AIH,

- akute oder „recent-onset AIH“ sowie

- AIH mit zentrilobulärer Nekrose.

Eine Leberschädigung mit Hepatitis und zentrilobulärer Nekrose sollte jedoch nur dann als AIH diagnostiziert werden, wenn alle anderen Kriterien vorliegen.

\section{Korrespondenzadresse}

\section{Prof. Dr. H.P. Dienes}

Institut für Pathologie,

Universitätsklinikum Köln,

Kompetenznetzwerk HepNet,

Deutsche Leberstiftung

Kerpener Str. 62, 50937 Köln

hans-peter.dienes@uk-koeln.de

Interessenkonflikt. Der korrespondierende Autor

gibt an, dass kein Interessenkonflikt besteht.

\section{Literatur}

1. Alvarez F, Berg PA, Bianchi FB et al. (1999) International Autoimmune Hepatitis Group Report: review of criteria for diagnosis of autoimmune hepatitis. J Hepatol 31: 929-938

2. Bedossa P, Moucari R, Chelbi E et al. (2007) Evidence for a role of nonalcoholic steatohepatitis in hepatitis C: a prospective study. Hepatology 46: 380-387

3. Brechot C, Thiers V, Kremsdorf D et al. (2001) Persistent hepatitis $B$ virus infection in subjects without hepatitis B surface antigen: clinically significant or purely "occult"? Hepatology 34: 194-203

4. Liu CJ, Chen PJ, Jeng YM et al. (2005) Serum adiponectin correlates with viral characteristics but not histologic features in patients with chronic hepatits. J Hepatol 54: 235-242

5. Drebber U, Kasper HU, Krupacz J et al. (2006) The role of Epstein-Barr virus in acute and chronic hepatitis. J Hepatol 44: 879-885

6. Fujita N, Sugimoto R et al. (2007) Hepcidin expression in the liver: relatively low level in patients with chronic hepatits C. Mol Med 13: 97-104

7. Hennes EM, Zeniya M, Czaja AJ et al. (2008) Simplified criteria for the diagnosis of autoimmune hepatitis. Hepatology 48: 169-176

8. Mifuji R, Kobayashi Y, Ma N et al. (2006) Role of transferrin receptor 2 in hepatic accumulation of iron in patients with chronic hepatitis C. J Gastroenterol Hepatol 21: 144-151

9. Misdraji J, Thiim M, Graeme-Cook FM (2004) Autoimmune hepatitis with centrilobular necrosis. Am J Surg Pathol 28: 471-478

10. Petit JM, Minello A, Jooste V et al. (2005) Decreased plasma adiponectin concentrations are closely related to steatosis in hepatitis C virus-infected patients. J Clin Endocrinol Metab 90: 2240-2243

11. Poussin K, Dienes H, Sirma H et al. (1999) Expression of mutated hepatitis $B$ virus $X$ genes in human hepatocellular carcinomas. Int J Cancer 80: 497505

12. Torbenson M, Thomas DL (2002) Occult hepatitis B. Lancet Infect Dis 2: 479-486

13. Valenti L, Pulixi EA, Arosio P et al. (2007) Relative contribution of iron genes, dysmetabolism and hepatitis $\mathrm{C}$ virus $(\mathrm{HCV})$ in the pathogenesis of altered iron regulation in HCV chronic hepatitis. Haematologica 92: 1037-1042

14. Yuki N, Nagaoka T, Yamashiro M et al. (2003) Longterm histologic and virologic outcomes of acute self-limited hepatitis B. Hepatology 37: 1172-1179

15. Zografos TA, Rigopoulou El, Liaskos C et al. (2006) Alterations of leptin during IFN-alpha therapy in patients with chronic viral hepatitis. J Hepatol 44: 848-855

16. Dienes HP, Drebber U, Both I von (1999) Liver biopsy in hepatitis C. J Hepatol 31: 43-46
R. Huss $\cdot$ X. Xiao $\cdot$ H. Heimberg

Diabetes Research Center, Vrije

Universiteit Brussel, Brüssel, Belgien

Adult stem cells regenerate the endocrine pancreas and normalize hyperglycaemia and insulin production in diabetic mice

\section{Verh Dtsch Ges Pathol (2005) 89: 184-190}

On behalf of the authors $\mathrm{H}$. Heimberg and $\mathrm{X}$. Xiao, the publication has been withdrawn since these authors had not given their consent to publication of this report, whose existence they were not aware of and whose content is in conflict with their data.

Im Auftrag der Autoren H. Heimberg und X. Xiao wird die oben genannte Publikation zurückgezogen, da diese Autoren weder von der Existenz dieses Beitrags wussten, noch seiner Veröffentlichung zugestimmt hatten. Darüber hinaus widersprechen ihre Ergebnisse dem Inhalt des Beitrags.

\section{Korrespondenzadresse}

Prof. Dr. H. Heimberg

Diabetes Research Center

Vrije Universiteit Brussel

Laarbeeklaan 103

1090 Brüssel, Belgien

Harry.Heimberg@vub.ac.be 